\title{
Multiparametric magnetic resonance imaging of the prostate at 1.5-Tesla without endorectal coil: Can it be used to detect clinically significant prostate cancer in men with medical devices that are contraindicated at 3-Tesla?
}

Jorge Abreu-Gomez ; Inga Isupov²; Matthew McInnes²; Trevor Flood²; Christopher Morash²; Nicola Schieda ${ }^{2}$

${ }^{1}$ Sunnybrook Health Sciences Centre, Toronto, ON, Canada; ${ }^{2}$ The Ottawa Hospital, Ottawa, ON, Canada

Cite as: Abreu-Gomez J, Isupov I, McInnes M, et al. Multiparametric magnetic resonance imaging of the prostate at 1.5-Tesla without endorectal coil: Can it be used to detect clinically significant prostate cancer in men with medical devices that are contraindicated at 3-Tesla? Can Urol Assoc J 2020 August 7; Epub ahead of print. http://dx.doi.org/10.5489/cuaj.6689

Published online August 7, 2020

$* * *$

\section{Introduction}

Multi-parametric (mp) MRI is accurate for detection of clinically significant (International Society of Urogenital Pathology [ISUP] grade group $\geq 2$ ) prostate cancer (CS-PCa) (1). Imaging quality is crucial for accurate mp-MRI and should be compliant with Prostate Imaging and Data Reporting System (PI-RADS) specifications $(2,3)$. mp-MRI can be performed without endorectal coil (ERC) at 3-Tesla (T) $(4,5)$ which improves patient tolerance, ease of use and imaging artifact (5). 1.5-Tesla mp-MRI without ERC is more controversial. PI-RADS version 2 suggests imaging at $3 \mathrm{~T}$ should be performed over $1.5 \mathrm{~T}$ whenever possible and ERC may be indispensable at $1.5 \mathrm{~T} \mathrm{(2);} \mathrm{however,} \mathrm{it} \mathrm{is} \mathrm{acknowledged} \mathrm{that} \mathrm{credible} \mathrm{results} \mathrm{have} \mathrm{been} \mathrm{obtained} \mathrm{at} 1.5 \mathrm{~T}$ without ERC (6). There remains a subset of men in whom 3T imaging is contraindicated; where 1.5T imaging is required, namely those with uncleared (MRI unsafe) medical devices for 3T. At institutions, such as our own, who now perform mp-MRI exclusively without ERC at 3T, accessibility and experience with ERC for $1.5 \mathrm{~T} \mathrm{mp-MRI} \mathrm{is} \mathrm{limited.} \mathrm{This} \mathrm{study} \mathrm{evaluates} \mathrm{the}$ accuracy for detecting CS-PCa using 1.5T mp-MRI without ERC in men with uncleared medical devices for $3 \mathrm{~T}$.

\section{Methods}

Through a quality-assurance waiver from the IRB, a search of our PACS identified 22 men with mp-MRI at 1.5T performed 2013-2018 due to uncleared medical devices for 3T: coronary stents or metallic cardiac graft/marker $\mathrm{N}=16$, metallic foreign body $\mathrm{N}=1$, iliac stent $\mathrm{N}=1$, aortic valve replacement $\mathrm{N}=2$ and unknown endoscopy clip $\mathrm{N}=1$. For a control group, 79 men with $3 \mathrm{~T}$ mpMRI performed during the same study period using a similar generation MRI system were 
identified. MRI were performed for AS or previous negative biopsy in all men with no MRI performed for biopsy naive patients. Figure 1 depicts patient inclusion/exclusion criteria.

\section{MRI technique}

MRI examinations were performed using 3T TRIO-TIM or 1.5T Symphony-TIM scanners (Siemens Medical, Malvern PA) using external phased-array coils [4 channels 3T, 12 channels $1.5 \mathrm{~T}]$ and integrated spine-array coils [3 channels $3 \mathrm{~T}, 6$ channels $1.5 \mathrm{~T}$ ] with the same operating software application (Siemens Syngo version MR B17, Malvern PA). ERC was not used. mpMRI protocols used at $1.5 \mathrm{~T}$ and $3 \mathrm{~T}$ are provided in Table 1.1.5T mp-MRI was matched, as closely as, possible to $3 \mathrm{~T}$, as described previously (7).

\section{Subjective interpretation of studies using PI-RADSv2}

A genitourinary radiologist with 14 years of experience in prostate MRI, the Director of Prostate Imaging at our institution (7), evaluated each examination blinded to patient clinical parameters and histopathology for the presence of tumor using PI-RADSv2 guidelines and sector map (2).

\section{Reference standard}

The reference standard was radical prostatectomy (RP). A genitourinary pathologist with 14 years of experience (BLINDED) reviewed the RP results. The dominant tumor foci within each $\mathrm{RP}$ (tumor foci measuring at least $0.5 \mathrm{~mL}$ in size) were identified and mapped to a prostate sector MRI-RP map (Figure 2). For all patients, there was a single dominant tumor focus.

\section{Statistical analysis}

Patient age, prostate serum antigen (PSA) and ISUP grade group were compared using independent t-tests and chi-square. Lesion size, pathological stage and lesion size was also compared using Wilcoxon Sign-Rank Test and Student's T test. Diagnostic accuracy of mp-MRI for detection of CS-PCa was tabulated. McNemar test was used to compare the detection rate of $\mathrm{CS}-\mathrm{PCa}$ at $1.5 \mathrm{~T}$ and $3 \mathrm{~T}$.

\section{Results}

Mean patient age and PSA were $69.2 \pm 7.6$ years and $8.5 \pm 6.5 \mathrm{ng} / \mathrm{mL}$ at $1.5 \mathrm{~T}$ and $63.4 \pm 5.2$ years and $8.4 \pm 5.2 \mathrm{ng} / \mathrm{mL}$ at $3 \mathrm{~T}$. Patients imaged at $1.5 \mathrm{~T}$ were older $(\mathrm{p}<0.001)$, with no difference in PSA ( $p=0.913)$. No difference in tumor size or clinical indication between groups was found $(\mathrm{p}>0.05)$. The $3 \mathrm{~T}$ group had higher rates of $\mathrm{pT} 3$ disease; however, only the former was significant (Table 2).

Distribution of PCa by ISUP grade group and PI-RADSv2 scores by field strength are summarized in Table 2. There was no difference in grade groups ( $\mathrm{p}=0.922)$. At $1.5 \mathrm{~T}, 85.7 \%$ $(18 / 21)$ of tumors were localized to the peripheral zone (PZ) and 14.3\% (3/21) the transition zone (TZ) at RP, compared to $89.9 \%(71 / 79) \mathrm{PZ}$ and $10.1 \%(8 / 79) \mathrm{TZ}$ at $3 \mathrm{~T},(\mathrm{p}=0.588)$. At $1.5 \mathrm{~T}$, the dominant tumor was accurately detected in $90.5 \%(19 / 21)$ compared to $93.7 \%(74 / 79)$ at $3 \mathrm{~T}$, 


\section{CUAJ - Research Letter \\ Abreu-Gomez et al \\ Detecting clinically significant PCa with mpMRI without endorectal coil}

$(\mathrm{p}=0.76)$. Diagnostic accuracy at $1.5 \mathrm{~T}$ and $3 \mathrm{~T}$ are summarized in Table 2.

\section{Discussion}

This study evaluated the accuracy of $1.5 \mathrm{~T} \mathrm{mp-MRI} \mathrm{performed} \mathrm{without} \mathrm{endorectal} \mathrm{coil} \mathrm{for}$ detection of CS-PCa in men with uncleared medical devices for imaging at 3T. Using similar generation $1.5 \mathrm{~T}$ and $3 \mathrm{~T}$ scanners from the same vendor with the same operating system, we demonstrated comparable accuracy at $1.5 \mathrm{~T}$ and $3 \mathrm{~T}$. Our results support the growing body of evidence suggesting that mp-MRI at $1.5 \mathrm{~T}$ for PCa detection may be an acceptable alternative to imaging at $3 \mathrm{~T}$ for patients with implanted devices uncleared for $3 \mathrm{~T}$ and, if more broadly applied, to patients who do not have reasonable access to 3T systems (7).

Ullrich et al. (6) demonstrated similar PI-RADS scores in patients imaged at 1.5T and 3T scanners. Similarly, Bratan et al. (8) and Thompson et al.(9) showed that MRI field strength and coil configuration had little influence on tumor detection rate. Moreover, a meta-analysis showed that ERC yielded no additional benefit for PCa detection accuracy or image quality at $1.5 \mathrm{~T}$ and $3 T(10)$.

Our study is limited by sample size, which can be expected given the single-center retrospective nature of our analysis. A proportion of the uncleared medical devices in our study group could now be considered relative rather than absolute contraindications for imaging at 3T. For example, many institutions (including at present, our own) no longer consider manufacturer recommendations for field strength when imaging coronary stents.

\section{Conclusions}

Our study demonstrates comparable accuracy of mp-MRI for detecting CS-PCa at both 1.5T and $3 \mathrm{~T}$ without endorectal coil and that non-ERC $1.5 \mathrm{~T} \mathrm{mp}-\mathrm{MRI}$ could be acceptable in men who cannot undergo 3T MRI due to an uncleared medical device and if more broadly applied, to those men who do not have access to $3 \mathrm{~T}$. 


\section{References}

1. Ahmed HU, El-Shater Bosaily A, Brown LC, Gabe R, Kaplan R, Parmar MK, et al. Diagnostic accuracy of multi-parametric MRI and TRUS biopsy in prostate cancer (PROMIS): a paired validating confirmatory study. Lancet. 2017;389(10071):815-22.

2. Weinreb JC, Barentsz JO, Choyke PL, Cornud F, Haider MA, Macura KJ, et al. PIRADS Prostate Imaging - Reporting and Data System: 2015, Version 2. Eur Urol [Internet]. 2016;69(1):16-40. Available from: http://dx.doi.org/10.1016/j.eururo.2015.08.052

3. Turkbey B, Rosenkrantz AB, Haider MA, Padhani AR, Villeirs G, Macura KJ, et al. Prostate Imaging Reporting and Data System Version 2.1: 2019 Update of Prostate Imaging Reporting and Data System Version 2. Eur Urol [Internet]. 2019;0232:1-12. Available from: https://linkinghub.elsevier.com/retrieve/pii/S0302283819301800

4. Gawlitza J, Reiss-Zimmermann M, Thormer G, Schaudinn A, Linder N, Garnov N, et al. Impact of the use of an endorectal coil for $3 \mathrm{~T}$ prostate MRI on image quality and cancer detection rate. Sci Rep. 2017 Feb;7:40640.

5. Heijmink SWTPJ, Futterer JJ, Hambrock T, Takahashi S, Scheenen TWJ, Huisman HJ, et al. Prostate cancer: body-array versus endorectal coil MR imaging at 3 T--comparison of image quality, localization, and staging performance. Radiology. 2007 Jul;244(1):18495.

6. Ullrich T, Quentin M, Oelers C, Dietzel F, Sawicki LM, Arsov C, et al. Magnetic resonance imaging of the prostate at 1.5 versus $3.0 \mathrm{~T}$ : A prospective comparison study of image quality. Eur J Radiol. 2017;90:192-7.

7. Abreu-Gomez J, Shabana W, McInnes MDF, O’Sullivan JP, Morash C, Schieda N. Regional Standardization of Prostate Multiparametric MRI Performance and Reporting: Is There a Role for a Director of Prostate Imaging? AJR Am J Roentgenol. 2019 Jun;1-7.

8. Bratan F, Niaf E, Melodelima C, Chesnais AL, Souchon R, Mege-Lechevallier F, et al. Influence of imaging and histological factors on prostate cancer detection and localisation on multiparametric MRI: a prospective study. Eur Radiol. 2013 Jul;23(7):2019-29.

9. Thompson JE, Moses D, Shnier R, Brenner P, Delprado W, Ponsky L, et al. Multiparametric magnetic resonance imaging guided diagnostic biopsy detects significant prostate cancer and could reduce unnecessary biopsies and over detection: a prospective study. J Urol. 2014 Jul;192(1):67-74.

10. Fusco R, Sansone M, Granata V, Setola SV, Petrillo A. A systematic review on multiparametric MR imaging in prostate cancer detection. Infect Agent Cancer. 2017;12:57. 


\section{Figures and Tables}

Fig. 1. Flow diagram shows patient selection used in this retrospective study. mpMRI: multiparametric magnetic resonance imaging; RP: radical prostatectomy.

1. Clinical 1.5T Tesla system: Symphony Tim (Siemens Syngo version MR B17, Malvern PA)

2. Discovery $750 \mathrm{~W}$ (General Electric, Milwaukee WI)

3. Clinical 3 Tesla system: TRIO Tim (Siemens Syngo version MR B17, Malvern PA)

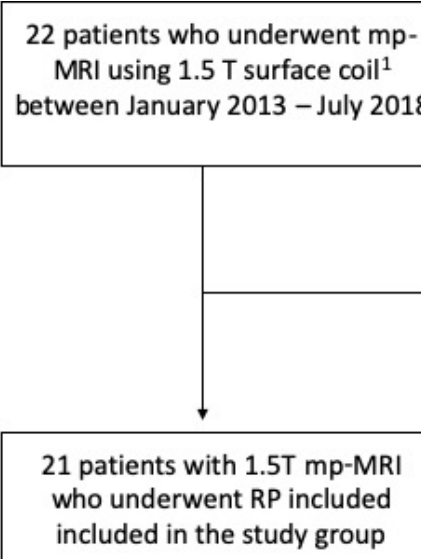

\section{Excluded due to: Radical prostatectomy not performed $(n=1)$}

Excluded due to: mp-MRI performed using alternate $3 \mathrm{~T}$ scanner $^{2}(n=250)$
329 patients with mp-MRI using 3T surface coil who underwent RP between January 2013 - July 2018

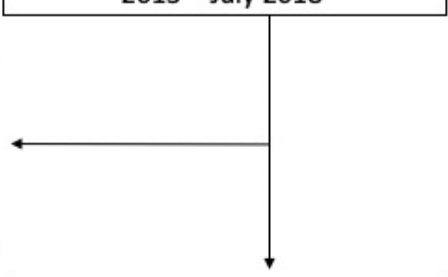

79 patients with $\mathrm{mp}-\mathrm{MRI}$ using comparable 3T scanner ${ }^{3}$ who underwent RP included in the control group




Fig. 2. 62-year old male with Gleason $3+4=7$ prostatic adenocarcinoma ( $\mathrm{PCa})$ in the right middle peripheral zone (PZ). Examination was obtained in a 1.5 T magnet. (A) Axial T2-weighted (T2W); (B) axial apparent diffusion coefficient (ADC) map; (C) echo-planar diffusion weighted image (DWI, $b=1000 \mathrm{~mm}^{2} / \mathrm{s}$ ); and (D) axial image from dynamic contrast enhanced (DCE) imaging with time-signal intensity (SI) curve show the tumor as a $11 \mathrm{~mm}$ hypointense focus (solid white arrow in A), which is mildly hypointense on ADC map (dotted arrow in B) and isointense to the adjacent parenchyma on high $\mathrm{b}$ value DWI (open white arrow in C). It was detectable as a focal enhancing nodule on DCE with a time-signal intensity (SI) curve within the tumor demonstrating type II kinetics. The tumor was considered PI-RADS v2 category 4 (DWI score 3 upgraded by DCE) observation. Corresponding low microscopic power (10x) hematoxylin and eosin (H\&E) stained photomicrograph $(\boldsymbol{E})$ shows the corresponding tumor outlined in black.

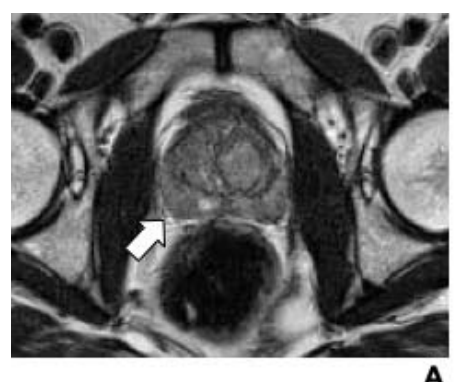



B
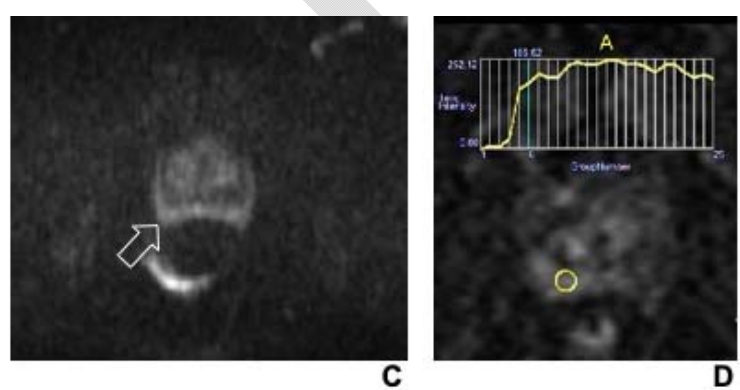

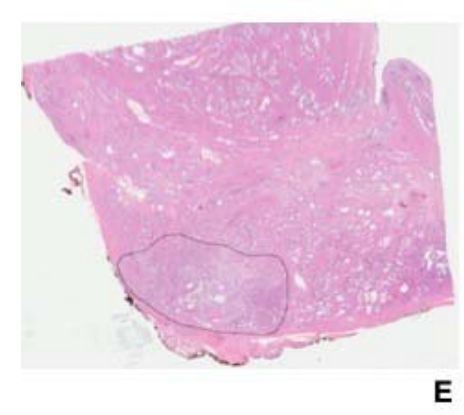


Detecting clinically significant PCa with mpMRI without endorectal coil

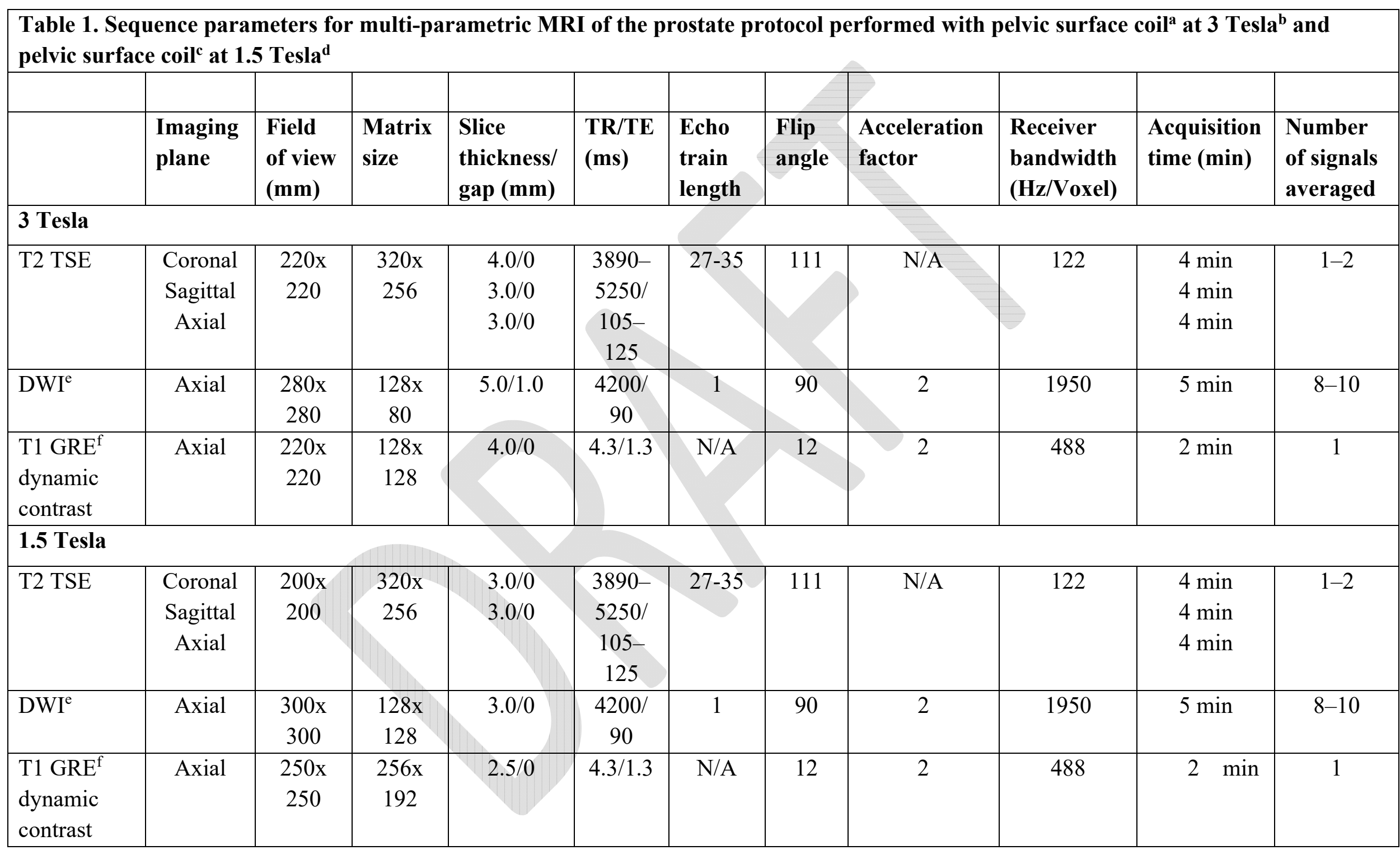




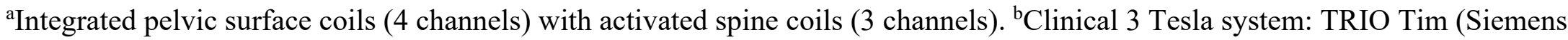
Healthcare). ${ }^{\mathrm{c}}$ Integrated pelvic surface coils (12 channels) with activated spine coils ( 6 channels). ${ }^{\mathrm{d}} \mathrm{Clinical} 1.5 \mathrm{~T}$ Tesla system: Symphony Tim (Siemens Healthcare). ${ }^{~} \mathrm{DWI}=$ Diffusion weighted imaging performed with spectral fat suppression echo planar imaging with tridirectional motion probing gradients and B values of $0,500,1000 \mathrm{~mm}^{2} / \mathrm{sec}$ with automatic apparent diffusion coefficient map generation. fDynamic fast spoiled 2D Gradient Recalled Echo performed with a temporal resolution of 10 seconds after injection of $0.1 \mathrm{mmol} / \mathrm{kg}$ of gadobutrol (Gadovist, Bayer Inc. Toronto, ON) at a rate of $3 \mathrm{~mL} / \mathrm{sec}$.

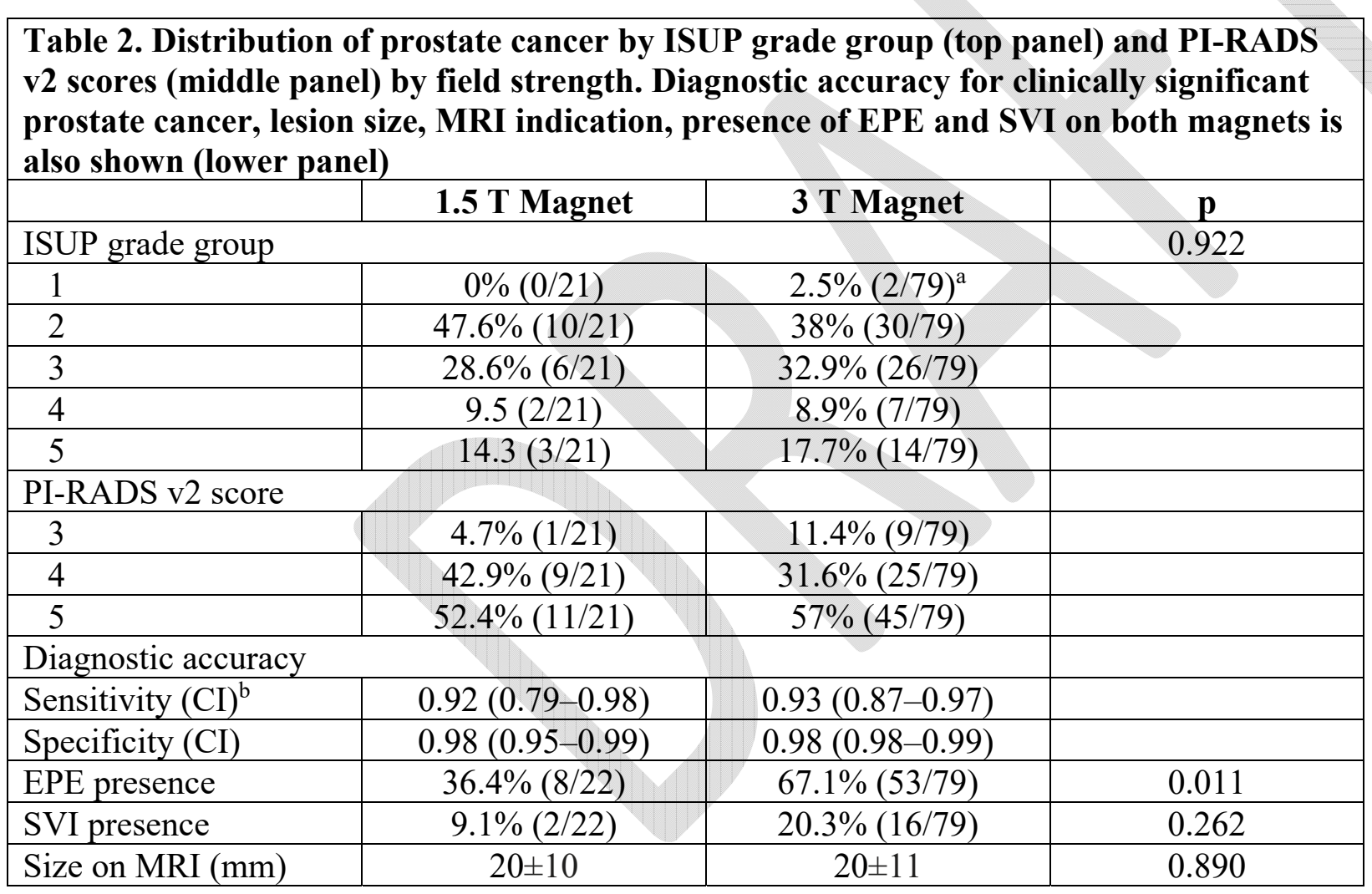


Detecting clinically significant PCa with mpMRI without endorectal coil

\begin{tabular}{|l|c|c|c|}
\hline MRI Indication & $\begin{array}{c}\text { Active surveillance } \\
\text { Previous negative } \\
\text { biopsy }\end{array}$ & $\begin{array}{c}\text { Active surveillance } \\
\text { Previous negative } \\
\text { biopsy }\end{array}$ & \\
\hline
\end{tabular}

${ }^{a}$ In this study, these 2 patients were counted as negative for clinically significant prostate cancer which was defined as ISUP $\geq 2$. b95\% Confidence interval. CI: confidence interval; EPE: extraprostatic extension; MRI: magnetic resonance imaging; SVI: seminal vesicle invasion. 